\title{
TRANSLUCENCY EVALUATION OF ZIRCONIA LITHIUM SILICATE AND LITHIUM DISILICATE GLASS CERAMIC WITH DIFFERENT THICKNESSES PRE AND POST CEMENTATION
}

\author{
Mahmoud Abdel Salam shakal* and Hany Aboulfotouh Abdelmohsen Oraby**
}

\begin{abstract}
OBJECTIVES: The aim of this study was to evaluate translucency of zirconia lithium silicate and lithium disilicate glass ceramic with different thicknesses, pre and post cementation.

MATERIALS AND METHODS: A 120 Specimens of the current study were fabricated out of the two tested materials each counting $\mathrm{N}=60$, A1 (translucent) shades of zirconia-reinforced lithium silicate glass ceramic (ZLS, Vita Suprinity, Vita Zahnfabrik, Germany) and A1(translucent) lithium disilicate glass ceramics (Rosetta SM, Korea). Specimens had a uniform rectangular shape with standard dimensions of $15 \mathrm{~mm}$ length and $10 \mathrm{~mm}$ width. Specimens of each material groups were distributed into three main groups based of specimen's thickness $0.5 \mathrm{~mm}, 1 \mathrm{~mm}, 1.5 \mathrm{~mm}$ each of these groups were further distributed into two subgroups based on translucency testing timing before or after cementation each counting 10 specimens. Specimens were fabricated by sectioning the ceramic blocks using a precision saw (IsoMet 4000, Buehler) under copious water coolant. Specimens final dimensions were confirmed with digital caliper. SPECTRO UV-Vis-Double BEAM spectrophotometer with an integrating sphere was used to evaluate the total transmittance of light as a percentage $\left(\mathrm{T}_{\mathrm{t}} \%\right)$ at a wavelength of $532 \mathrm{~nm}$ for comparison among groups.
\end{abstract}

RESULTS: In the present study, translucency values at $0.5 \mathrm{~mm}$ and $1 \mathrm{~mm}$ thicknesses LDS were higher than those recorded for ZLS at the same thicknesses. While with $1.5 \mathrm{~mm}$ thick specimens the translucencies of both ZLS and LDS materials were fairly similar.

CONCLUSIONS: From the outcome of the current study, the following conclusions could be drawn out 1 . The thinner the ceramic veneering layer, the greater will be the impact of the underlying shade on the final shade of the restoration. 2. The translucency values recorded for the translucent zirconia materials were not only almost close to those of the lithium disilicate ceramic material, but also comparable to the translucency values reported for enamel or dentin of similar thicknesses.

KEYWORDS: Vita Suprinity, Rosetta SM, UV-Vis spectrophotometer. * Assistant Professor of Fixed Prosthodontics, Department of fixed prosthodontics, Faculty of Dentistry, Tanta University, Egypt
** Lecturer of fixed prosthodontics, Department of fixed prosthodontics, Faculty of Dentistry, Tanta university, Egypt 


\section{INTRODUCTION}

The increasing awareness of patients toward aesthetic restorative materials and techniques that resembles the natural tooth', have resulted in the development of a wide variety of techniques including and not limited to laminates, inlays, onlays and full coverage crowns and new all ceramic systems. ${ }^{[1,2]}$. Replication of the natural tooth shape, size, translucency and surface texture is an essential part of acquiring an aesthetically pleasant restoration ${ }^{[3]}$. Opacity caused by the metal substructure and opaquer layer in Porcelain fused to metal restoration has compromised the natural appearance and affect the overall esthetic result of the restoration despite of the clinical success that was offered over years. ${ }^{[4]}$

Perception of color is a response of human visual system to the light reflected from objects based on psychophysical elements. ${ }^{[5]}$ One of the elements that has the greatest share in patient's satisfaction for their restoration especially in the anterior region is color. ${ }^{[6]}$ The high aesthetic quality of porcelain laminate veneering because of the combination of high aesthetic appeal, patient satisfaction, and less-invasive tooth preparation has led to the vast increasing interest in their use as aesthetic restorations ${ }^{[7]}$.

Factors such as crystalline structure, grain size, pigments and the size, number and distribution of defects porosity as well, controls and determine the degree of dental ceramics translucency, or in another word if the ceramic crystals are smaller than the wavelength of visible light, it appear transparent. While with bigger crystals ceramics light scattering and diffuse reflection occurs and the ceramic would appear opaque. Light Scattering within the body attenuates the emergent beam intensity most effectively within the body of the ceramic, causing portion of the light may be absorbed ${ }^{[8]}$.

The development of new all-ceramic systems with a deeper translucency similar to natural tooth to satisfy the patients need, is considered one of the main factors in controlling the final restoration esthetics. Zirconia-based ceramics, is among these new materials that fulfill the biomechanical requirements; such as dimensional and chemical stability, high fracture toughness and mechanical strength and therefore are considered to be very attractive for dentists. Increasing the crystalline content of all-ceramic materials respectively resulted not only in increase in their strength, but also increases its opacity as well due to differences in homogeneity of the crystals and the refractive indices. Compared to many all-ceramic systems zirconia cores are of poor translucency and extremely white in esthetic appearance. [9,10] Successful attempts were made to improve the optical characteristics of these restorations with the development of shaded zirconia cores, that has resulted in better transmission of light and improving the reproduction of color and translucency in relation to the natural teeth ${ }^{[11]}$.

Translucency, opalescence, fluorescence, surface texture and shape properties, the brand and batches of the porcelain used, the number of porcelain firings and the condensation technique and also by the color, translucency, and the thickness of the underlying resin luting agent are factors that contribute in the final ceramic restoration color ${ }^{[12]}$. Efforts are made by the manufacturers to accurately predicts the final color of restorations, offering a wide variety of colors available for resin lutingagent shades. Both the type, the shade of resin cement and the thickness and shade of the ceramic influence the resulting optical color of laminate restorations when a colorimeter is used before and after bonding as reported recently ${ }^{[13]}$.

The finally perceived tooth color is a combination of the light reflected from the tooth surface with that redirected from dentin, which results from internal reflection and refraction. ${ }^{[14-15]}$. Furthermore, color has three dimensions: chroma, hue, and value. However, when replication of tooth with an esthetic 
restoration is aimed, translucency is considered an important factor that should be considered, or in another ward the extent to which light is diffused rather than absorbed or reflected ${ }^{[16]}$ as presented with the high translucent enamel at the incisal third and the proximal surfaces of a tooth. ${ }^{[17]}$ Whereas, the middle third enamel is affected markedly by the yellowish dentin. ${ }^{[18]}$

The inherent translucency properties of the all ceramic, allows dentists and laboratory technicians to fabricate restorations that are similar to natural teeth, which is considered to be a critical factor in producing a restoration with improved esthetics. The optical and strength properties of the restoration is greatly affected by their compositions, microstructures, crystalline contents, and phases, which is different from one material to another. These ceramic systems can be divided into glasscontaining materials (such as feldspathic porcelain), reinforced-glass materials (such as leucite and lithium disilicate), glass-infiltrated crystalline materials, and purely crystalline materials (such as zirconia and alumina). However, an increase in the crystalline content to achieve greater strength often results in greater opacity ${ }^{[19]}$.

The minimally invasive tooth preparation technique results consequently in reduction of the veneering restorative material thickness and consequently will leads to increased translucency of the veneering layer that may compromise the target aesthetic quality of the final restoration unless the factors related to the cementing technique used are precisely controlled. Therefore, this study was directed toward evaluating the translucency of zirconia lithium silicate and lithium disilicate glass ceramic with different thicknesses, pre and post cementation.

\section{AIM OF THE WORK}

The aim of this study was to evaluate translucency of zirconia lithium silicate and lithium disilicate glass ceramic with different thicknesses, pre and post cementation.

\section{MATERIALS AND METHODS}

A 120 Specimens were fabricated out of the two tested materials each counting 60, A1 (translucent) shades of zirconia-reinforced lithium silicate glass ceramic (ZLS, Vita Suprinity, Vita Zahnfabrik, Germany) and A1(translucent) lithium disilicate glass ceramics (LDS, Rosetta SM, Korea). Specimens had a uniform rectangular shape with standard dimensions of $15 \mathrm{~mm}$ length and 10 $\mathrm{mm}$ width. Specimens of each material groups were distributed into three main groups each counting 20 based of specimen's thickness $0.5 \mathrm{~mm}$, $1 \mathrm{~mm}, 1.5 \mathrm{~mm}$, each of these groups were further distributed into two subgroups each counting 10 based on translucency testing timing before or after cementation. Specimen grouping and distribution are shown in table (1).

Specimens were fabricated by sectioning the ceramic blocks using a precision saw (IsoMet 4000, Buehler) under copious water coolant. Specimens final dimensions were confirmed with digital caliper. (Fig.1) All sectioned specimens were sintered according to the manufacturer instructions in a high temperature furnace (Ivoclar Vivadent Programat). The specimens were cleaned in ultrasonic water path before translucency testing with the spectrophotometry.

\section{Translucency testing}

Spectrophotometer is commonly used for the quantitative measurement of the reflection or transmission properties of opaque solids or transparent material as a function of wave. It is more specific as it deals with near-ultraviolet, visible light and infrared. Spectrophotometer compares the light intensity differences between two light paths, one path containing the test sample and the other is the reference sample. Values of each wave length representing transmittance or reflectance of the test sample is then compared with those for the reference sample. Specimens translucency was calculated by 
TABLE (1) Specimens grouping and distribution

\begin{tabular}{|c|c|c|c|c|c|c|}
\hline Parameter & \multicolumn{3}{|c|}{ ZLS (N=60) } & \multicolumn{3}{c|}{ LDS (N=60) } \\
\hline Thickness & $\begin{array}{c}0.5 \mathrm{~mm} \\
(\mathrm{~N}=20)\end{array}$ & $\begin{array}{c}1 \mathrm{~mm} \\
(\mathrm{~N}=20)\end{array}$ & $\begin{array}{c}1.5 \mathrm{~mm} \\
(\mathrm{~N}=20)\end{array}$ & $\begin{array}{c}0.5 \mathrm{~mm} \\
(\mathrm{~N}=20)\end{array}$ & $\begin{array}{c}1 \mathrm{~mm} \\
(\mathrm{~N}=20)\end{array}$ & $\begin{array}{c}1.5 \mathrm{~mm} \\
(\mathrm{~N}=20)\end{array}$ \\
\hline Pre-cementation & $(\mathrm{N}=10)$ & $(\mathrm{N}=10)$ & $(\mathrm{N}=10)$ & $(\mathrm{N}=10)$ & $(\mathrm{N}=10)$ & $(\mathrm{N}=10)$ \\
\hline Post cementation & $(\mathrm{N}=10)$ & $(\mathrm{N}=10)$ & $(\mathrm{N}=10)$ & $(\mathrm{N}=10)$ & $(\mathrm{N}=10)$ & $(\mathrm{N}=10)$ \\
\hline
\end{tabular}

the following logarithmic equation $\left(A=2-\log _{10} \%\right.$ $\boldsymbol{T})$, where (A) is representing absorbance ratio of the specimen and $(\mathrm{T})$ is representing transmittance ratio.

\section{Pre-cementation Translucency testing protocol}

Specimens translucency of the pre-cementation group of both materials were measured under standardized protocol for color evaluation adopted for all discs. All readings were carried out in the same room under standardized lighting conditions. Spectrophotometer (SPECTRO UV-Vis-Double BEAM PC SCANING SPECTROPHOTOMETER UVD2950) with an integrating sphere was used to evaluate the total transmittance of light as a percentage $\left(\mathrm{T}_{\mathrm{t}} \%\right)$ at a wavelength of $532 \mathrm{~nm}$ for comparison among groups. (Fig. 2)

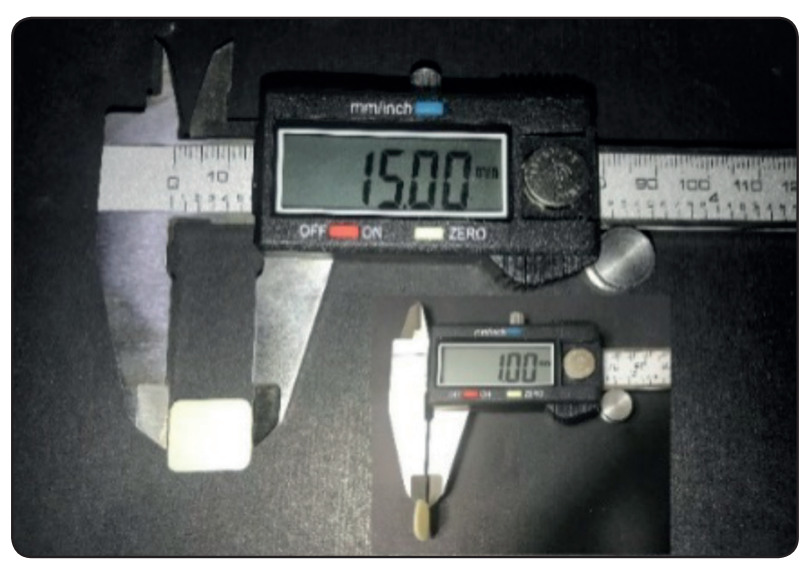

Fig. (1) Specimens measurements confirmation

\section{Specimens cementation protocol}

Post-cementation group of specimens were luted with light cured resin cement (Choice 2 Veneer, BISCO USA) translucent shade. The luting resin cement was applied on the ceramic discs specimens and pressed against a clean glass plate surface. A digital veneer caliper was used to make sure a standardized cement thickness of $0.05 \mathrm{~mm}$ between the glass plate and the ceramic disc, then light cured for $60 \mathrm{~s}$.

\section{Post-cementation Translucency testing protocol}

Translucency testing of the specimens of this group were carried out with the same parameters and condition used to measure translucency of the pre-cementation group of specimens.

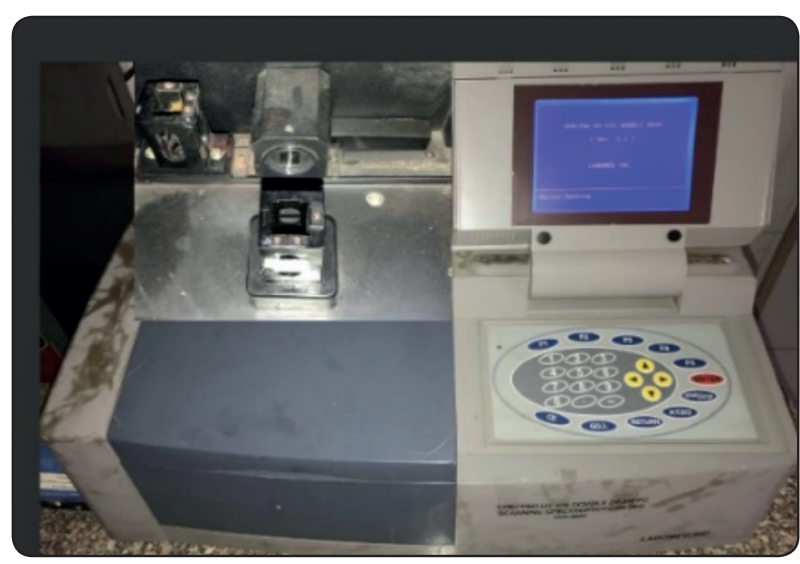

Fig. (2) Specimens measurements translucency measurements in spectrophotometer. 


\section{RESULTS}

Speciemenns translucenecy were calcultaed with the formerly metioned equation $\left(A=2-\log _{10} \%\right.$ $T)$. Measurements were collected and Statistically analyzed using SPSS software program (Statistical Package for the Social Science; SPSS, Chicago, Illinois, USA) version23 for Microsoft Windows.

The statistical data are reported as the mean and standard deviation (SD), A comparison of numerical variables between the study groups was performed using Student's t-test to compare independent samples from two groups when the samples were normally distributed, Paired t-test used to compare paired samples from two groups when the samples were normally $\mathrm{P}$-value less than 0.05 (two-tailed) were considered statistically significant. Outcome measures were analyzed using Student t-test, paired t-test, ANOVA and post hoc multiple comparisons. The mean values, Standard deviation of specimen's transparency are listed in (Table. 2) and statistical significance graphically represented in (Figure.3, 4 and 5).

Paired test was used to evaluate significant difference in values obtained pre and post- cementation for both tested materials LDS and ZLS in different thickness. There was significant difference seen between both pre and post cementation groups within the LDS group of thicknesses $(0.5 \mathrm{~mm}, 1 \mathrm{~mm})$ ( $\mathrm{P}$-value $=0.016$ and 0.003 respectively), while there was no sufficient evidence of significant difference between pre and post cementation groups with LDS specimen thicknesses $1.5 \mathrm{~mm}$ (p-value $=0.403$ ). In the other side, there was also significant difference between pre and post cementation of ZLS $1 \mathrm{~mm}$ (Pvalue $=0.024)$, while there was no significant difference between pre and post cementation values recoded for ZLS specimen at thicknesses of 0.5 $\mathrm{mm}$ and $1.5 \mathrm{~mm}$ respectively at the level of (P-value $=0.084$ and 0.177 respectively)

ANOVA test showed significant difference between values obtained with LDS at different thicknesses of $(0.5 \mathrm{~mm} 1 \mathrm{~mm} 1.5 \mathrm{~mm})(\mathrm{P}$-value $<0.001)$. Post Hoc multiple comparison test_using Tukey test showed significant difference between values obtained post cementation with the LDS thickness of $0.5 \mathrm{~mm}$ and $1 \mathrm{~mm}$ (p-value $<0.001$ ) and between at thickness $0.5 \mathrm{~mm}$ and $1.5 \mathrm{~mm}(\mathrm{p}$-value $<0.001)$ and also there was sufficient evidence that there is significant difference between post cementation values with LDS thickness $1 \mathrm{~mm}$ and $1.5 \mathrm{~mm}$ ( $p$-value=0.0.01). Also, ANOVA test showed significant difference between post cementation values obtained with ZLS at specimen's thicknesses of $(0.5 \mathrm{~mm} 1 \mathrm{~mm} 1.5 \mathrm{~mm})$ (P-value $<0.001)$. Post Hoc multiple comparison: using Tukey test showed significant difference between values obtained with the post cementation ZLS at thicknesses of $0.5 \mathrm{~mm}$ and $1 \mathrm{~mm}$ (p-value<0.001) and between values

TABLE (2) Mean and standard deviation of the specimen's translucency

\begin{tabular}{|l|c|c|c|c|c|c|}
\hline Specimens type & \multicolumn{3}{|c|}{ LDS (N=60) } & \multicolumn{3}{c|}{ ZLS (N=60) } \\
\hline Specimens size & $\begin{array}{c}20 \\
\text { specimens }\end{array}$ & $\begin{array}{c}20 \\
\text { specimens }\end{array}$ & $\begin{array}{c}20 \\
\text { specimens }\end{array}$ & $\begin{array}{c}20 \\
\text { specimens }\end{array}$ & $\begin{array}{c}20 \\
\text { specimens }\end{array}$ & $\begin{array}{c}20 \\
\text { specimens }\end{array}$ \\
\hline Specimen Thickness & $0.5 \mathrm{~mm}$ & $1 \mathrm{~mm}$ & $1.5 \mathrm{~mm}$ & $0.5 \mathrm{~mm}$ & $1 \mathrm{~mm}$ & $1.5 \mathrm{~mm}$ \\
\hline Pre-cementation Mean values & 2.600 & 1.779 & 1.160 & 2.255 & 1.512 & 1.110 \\
\hline Standard deviation & \pm 0.226 & \pm 0.253 & \pm 0.151 & \pm 0.144 & \pm 0.277 & \pm 0.124 \\
\hline Post-cementation Mean values & 2.317 & 1.3940 & 1.057 & 2.075 & 1.180 & 0.987 \\
\hline Standard Deviation & \pm 0.198 & \pm 0.0892 & \pm 0.349 & \pm 0.198 & \pm 0.223 & \pm 0.231 \\
\hline
\end{tabular}


obtained with ZLS at thicknesses of $0.5 \mathrm{~mm}$ and $1.5 \mathrm{~mm}$ (p-value $<0.001$ ), but there was no sufficient evidence that there is significant difference between post cementation values at ZLS thicknesses of $1 \mathrm{~mm}$ and $1.5 \mathrm{~mm}$ (p-value $<0.136$ ). Statistical analysis outcome using the student $\mathrm{T}$ Test showed significant difference between values obtained with LDS and those of ZLS at thickness $0.5 \mathrm{~mm}(\mathrm{p}$-value $=0.014)$ post cementation, also a significant difference between LDS and those of ZLS at thickness of $1 \mathrm{~mm}$ ( $\mathrm{p}$-value $=0.011$ ), while there was no significant difference between LDS and those of ZLS at thickness value of $1.5 \mathrm{~mm}$ ( $\mathrm{p}$-value $=0.603$ )

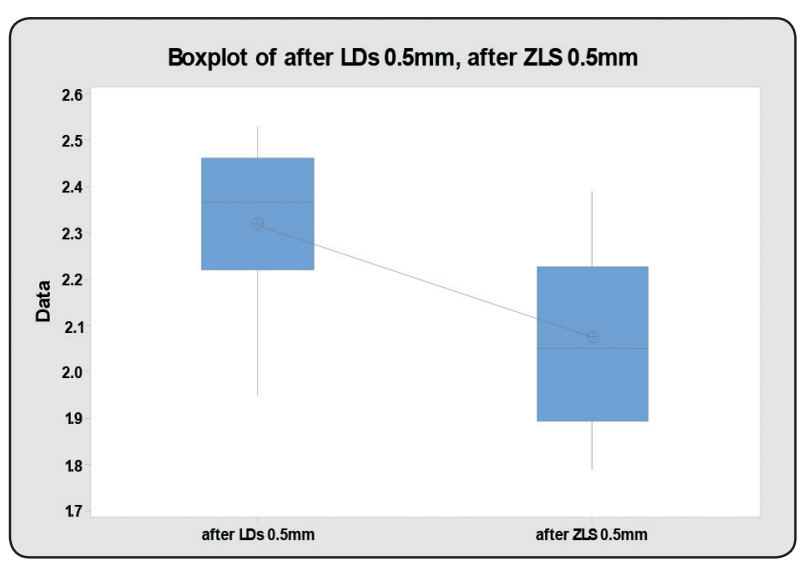

Fig. (3) Significant difference graphical representation of 0.5 $\mathrm{mm}$ thick specimens at $\mathrm{p}$-value $=0.014$

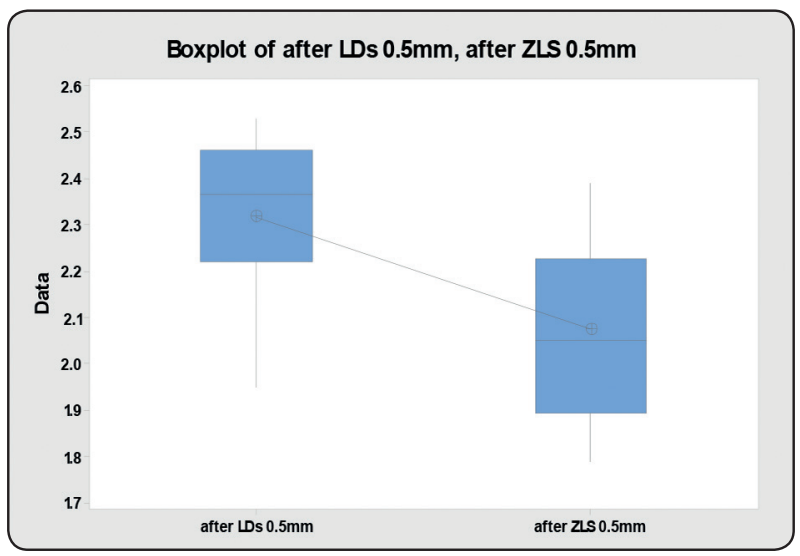

Fig. (4) Significant difference graphical representation of $1 \mathrm{~mm}$ thick specimens at $(\mathrm{p}$-value $=$ value $=0.011$

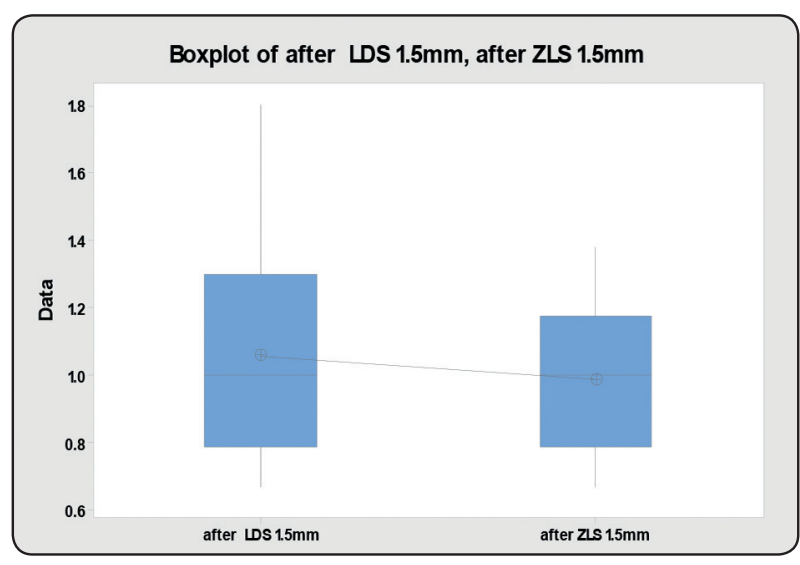

Fig. (5) Non-Significance difference graphical representation of $1.5 \mathrm{~mm}$ thick specimens at $\mathrm{P}$-value $=0.603$

\section{DISCUSSION}

Translucency of the restorative material is one of the stone corners in aesthetic success of a prosthetic restoration when it is considered. Other factors such as underlying tooth structure, cement opacity and shade, necessary thickness of the restoration, and the location of the tooth in the arch to be restored must be taken into consideration. Therefore, acquaintance with the material nature and optical property in regard the degree of its translucency allows the dentist to fabricate a natural-looking, esthetic restorations that mimic the natural blend between the dentine opacity and the relative enamel translucency ${ }^{(21-22)}$.

Translucency and light diffusion property of Lithium disilicate ceramic material and its capability to replicate natural tooth structure for esthetic undetectable restoration is one of the reasons that made them a material of choice for superior esthetics restoration. Recently, a new addition to the glass ceramics is the zirconia reinforced lithium silicate. This combination was made for the purpose of combining both zirconia and glass ceramics material characteristics as reported in an initial in vitro testing of the ZLS, however these materials are new to the market ${ }^{(23-24)}$.

Translucency of the tested materials in the current study has significantly decreased for both ceramic materials with each incremental increase in 
specimen's thickness, that goes in accordance with other studies, who reported the significant decrease in the translucency parameter of ceramics materials with increasing specimens the thicknesses ${ }^{(25-26)}$.

The lowered translucency parameter with increasing specimens thickness could be attributed to the lowered translucency values for all ceramic specimens, causing increased masking ability with 1.5-mm-thick discs. These results are in agreement with those reported by Vichi, where translucency values decreased with increasing ceramic thickness from 1.0 to $1.5 \mathrm{~mm}$. The results of the current study match the outcome of Hilgert study, who also reported the lowered translucency values with increasing ceramic veneers thickness from 0.4 to 0.7 and $1.0 \mathrm{~mm}^{(27-28)}$.

The relative comparable translucency of zirconia reinforced lithium silicate glass-ceramic could be attributed to the homogenous crystalline structure and finer crystal size $(0.5 \mu \mathrm{m})$ compared to the needle-shaped coarser crystalline structure $(1.5 \mu \mathrm{m})$ of lithium silicate glass as a result of the addition of zirconia and the ensuing nucleation process as explained by Heffernan et al, ${ }^{(29)}$ who related the discrepancies in the amount of reflected, absorbed and transmitted light is dependent to several factors including the particles' size compared to the incident light's wavelength $(0.4-0.7 \mu \mathrm{m})$ based on his findings of the relation between the increase of porcelain translucency to the decreased particles size in its composition, irregularities in the distribution of the phases, and the optical anisotropy of the grains. However, the results of the current study were not consistent with Giordano, who related the increased opacity of the glass ceramics to the volume of its glass content, which is against the fact of higher glass content in IPS e.max CAD (57-80\%) than in the case of Vita Suprinity (56-64\%) before crystallization, which is supposed to be increased after crystallization, so the former will still have higher glass content than the latter, however that point of arguments needs further investigations ${ }^{(30,31)}$.
The high translucent values of ZLS materials recorded in the current study shown to be relatively comparable with lithium disilicate translucency at clinically recommended thicknesses with the added mechanical strength because of zirconium addition at similar thicknesses, which indicates that restorations using these materials may have a promising future ${ }^{(32)}$. The results of the current study are also in accordance with other studies reporting that ceramic veneering of $1 \mathrm{~mm}$ thickness or less will be affected greatly by the shade of the core and cement as a result of translucency of all ceramic crowns. ${ }^{(33-35)}$

\section{CONCLUSION}

From the outcome of the current study, the following conclusions could be drawn out

1. The thinner the ceramic veneering layer, the greater will be the impact of the underlying shade on the final shade of the restoration.

2. The translucency values recorded for the translucent zirconia materials were not only relatively comparable to those of the lithium disilicate ceramic material, but also to the translucency index reported for enamel or dentin of similar thicknesses.

\section{REFERENCES}

1. Raigrodski AJ. Contemporary materials and technologies for all-ceramic fixed partial dentures: a review of the literature. J Prosthet Dent. 2004;92:557-62.

2. Conrad HJ, Seong WJ, Pesun IJ. Current ceramic materials and systems with clinical recommendations: a systematic review. J Prosthet Dent. 2007;98:389-404.

3. Rosenstiel SF, Land MF, Fujimoto J. Contemporary fixed prosthodontics.St. Louis, Mo:Mosby Elsevier.2006,p. 774.

4. Raptis NV, Michalakis KX, Hirayama H. Optical behavior of current ceramic systems. Int J Periodontics Restorative Dent. 2006;26:31-41.

5. Phira Sithiamnuai, Trinuch Eiampongpaiboon, Arati Shrestha, Kallaya Suputtamongkol. The effect of thickness on the contrast ratio and color of veneering ceramics. M Dent J. 2014;34(2):137-43. 
6- Gómez-Polo, C., Gómez-Polo, M., Celemín-Viñuela, A., Martínez Vázquez De Parga, J.A., 2014. Differences between the human eye and the spectrophotometer in the shade matching of tooth colour. J. Dent. 42, 742-5

7- Calamia, J.R., Calamia, C.S., 2007. Porcelain laminate veneers: reasons for 25 years of success. Dent Clin North Am 51, 399-417.

8. Van Noort, R. Introduction to Dental Materials, 4th ed.; Elsevier: Amsterdam, The Netherlands, 2013; pp. 231-246.

9. Heffernan MJ, Aquilino SA, Diaz-Arnold AM, Haselton DR, Stanford CM, Vargas MA. Relative translucency of six all-ceramic systems. Part I: core materials. J Prosthet Dent. 2002;88:4-9.

10. Baldissara P, Llukacej A, Ciocca L, Valandro FL, Scotti R. Translucency of zirconia copings made with different CAD/ CAM systems. J Prosthet Dent. 2010;104:6-12.

11. Christensen GJ. Esthetic dentistry-2008. Alpha Omegan. 2008;101:69-70.

12- Dozic, A., Kleverlaan, C.J., Meegdes, M., van der Zel, J., Feilzer, A.J., 2003. The influence of porcelain layer thickness on the final shade of ceramic restorations. J. Prosthet. Dent. 90, 563-570.

13- Turgut, S., Bagis, B., 2013. Effect of resin cement and ceramic thickness on final color of laminate veneers: an in vitro study. J. Prosthet. Dent. 109, 179-86.

14. The glossary of prosthodontic terms. The academy of prosthodontics. J Prosthet Dent. 1994;71:41-

15. Lemire PA, Burk B: Color in Dentistry. Hartford, CO, J.M. Ney Co., 1975. 16. Chu SJ, Devigus A, Mieleszko AJ: Fundamentals of Color: Shade Matching and Communication in Esthetic Dentistry. Chicago, IL, Quintessence Pub. Co., 2004.

17. McLean JW: The Science and Art of Dental Ceramics: A Collection of Monographs. Baton Rouge, LA, Louisiana State University School of Dentistry Continuing Education Program, 1974.

18. McLaren EA, Cao PT: Ceramics in dentistry-part I: classes of materials. Inside Dent. 2009;5:94-104.

19- Kim MJ, Ahn JS, Kim JH, Kim HY, Kim WC. Effects of the sintering conditions of dental zirconia ceramics on the grain size and translucency. J Adv Prosthodont. 2013;5(2):161-166.

20- Yu B, Ahn JS, Lee YK. Measurement of translucency of tooth enamel and detin. Acta Odontol Scand. 2009;67(1):57-64.

21. Della Bona A, Nogueira AD, Pecho OE. Optical properties of CAD-CAM ceramic systems. J Dent. 2014;42(9):12021209.
22. Sulaiman TA, Abdulmajeed AA, Donovan TE, et al. Optical properties and light irradiance of monolithic zirconia at variable thicknesses. Dent Mater. 2015;31(10):1180-1187.

23. Kojic DD, Singhal S, Shah S. CAD/CAM ceramic crown retention of resin cements. J Biotechnol Biomater. 2014;4(1):1-7.

24. Gracis S, Thompson V, Ferencz J et al. A new classification system for all-ceramic and ceramic-like restorative materials. Int J Prosthodont. 2015;28:227-235.

25. Bunek SS, Baumann A, Yapp R, Paravina RD, Powers JM. Translucency of CAD/CAM ceramics [The Dental Advisor Research Report 59]. May 2014. http://www.bruxzir. com/downloads/ Translucency-of-CAD-CAM-Ceramics. pdf. Accessed November 17, 2016.

26. Sulaiman TA, Abdulmajeed AA, Donovan TE, et al. Optical properties and light irradiance of monolithic zirconia at variable thicknesses. Dent Mater. 2015;31(10):1180-1187.

27. Vichi A, Ferrari M, \& Davidson CL. Influence of ceramic and cement thickness on the masking of various types of opaque posts Journal of Prosthetic Dentistry. 2000;83(4):412-417.

28. Hilgert LA, Araujo E, Baratieri LN, Edelhoff D, \& Gernet W. Influence of stump shade, ceramic thickness and translucency on the color of veneers Dental Materials. 2009;25(5) e9.

29- Heffernan MJ, Aquilino SA, Diaz-Arnold AM, Haselton DR, Stanford CM, Vargas MA. Relative translucency of six all-ceramic systems. Part I: core materials. J Prosthet Dent. 2002;88:4-9.

30- Giordano RA. Dental ceramic restorative systems. Compend Contin Educ Dent. 1996;17:779-86.

31- VITA Zahnfabrik. Vita Suprinity Technical and scientific documentation. 2014.

32- CHURCH T. Translucency and Strength of High Translucency Monolithic Zirconium Oxide Materials. MED SAN ANT U.S; 2016;81:11-17.

33. Li Q, Yu H, Wang YN. Spectrophotometric evaluation of the optical influence of core build up composites on all ceramic materials. Dental Materials. 2009;25:158-165.

34. Jarad FD, Moss BW, Youngson CC, Russell MD. The effect of porcelain thickness on color and the ability of a shade guide to prescribe chroma. Dental Materials. 2007;23: 456-460.

35. Chang J, Da Silva, Sakai M, Kristiansen J, Nagai SI. The optical effect of composite luting cement on all ceramic crowns. Journal of Dentistry. 2009;37:937-943. 